\title{
On the Reduction of Singular Matrix Pencils
}

\author{
By H. W. Turnbull. \\ (Received 13th March, 1934. Read 7th December, 1934.)
}

\section{Introduction.}

The following rational method of dealing with the reduction of a singular matrix pencil to canonical form has certain advantages. It is based on the principle of vector chains, the length of the chain determining a minimal index. This treatment is analogous to that employed by $\mathrm{Dr}$ A. C. Aitken and the author in Canonical Matrices (1932) 45-57, for the nonsingular case. In Theorems 1 and 2 tests are explicitly given for determining the minimal indices. Theorem 2 gives a method of discovering the lowest row (or column) minimal index. Theoretically it should be possible to state a corresponding theorem for each of these indices, not necessarily the lowest, and prior to any reduction of the pencil. This extension still awaits solution.

Theorem 3 is logically equivalent to the arguments used by Kronecker (who was the first to discuss the singular case, Berlin Sitzungsberichte (1890), 1375 and (1891), 9, 33) and subsequently by Dickson (Trans. American Math. Soc., 29 (1927), 239-253). For a geometrical treatment see Segre, Atti Acc. Torino, 19 (1 884), 878.

§. Let

$$
\Lambda=r A+s B=\left[r a_{i j}+s b_{i j}\right]
$$

be a matrix pencil, where the elements $a_{i j}$ and $b_{i j}$ all belong to a field $\mathcal{F}$, while $r, s$ are independent variables. Each matrix $A, B, \Lambda$ is assumed to have $n$ rows and $n^{\prime}$ columns, while neither $A$ nor $B$ is a scalar multiple of the other. It is proposed to reduce $\Lambda$ to a canonical form

$$
P . Q Q, \quad|P| \neq 0, \quad|Q| \neq 0,
$$

where $P$ and $Q$ are nonsingular constant matrices with elements in $\mathcal{F}$, $P$ having $n$ rows and columns, and $Q$ having $n^{\prime}$.

Let $\rho$ be the rank of $\Lambda$ in $r$ and $s$ : that is, let $\rho$ be the highest order among the minor determinants of $\Lambda$ which do not vanish 
identically for all $r$ and $s$. Then obviously there exist nonnegative integers $\mu$ and $\mu^{\prime}$ such that

$$
\mu=n-\rho \geqq 0, \quad \mu^{\prime}=n^{\prime}-\rho \geqq 0 .
$$

Two cases arise, the singular and the nonsingular. In the latter both $\mu$ and $\mu^{\prime}$ are zero: in the former at least one, $\mu$ say, is nonzero. It will be proved that in the singular case $\mu$ is the number of ways, linearly independent in $\mathcal{F}$, in which the rows of $\Lambda$ are linearly related, while $\mu^{\prime}$ similarly relates the columns.

Such a row relation $\sum_{i=1}^{n} \theta_{i}$ row $_{i}=0$ can be conveniently written as a matrix product

$$
\theta \Lambda \equiv\left[\theta_{1}, \theta_{2}, \ldots, \theta_{n}\right](r A+s B)=0
$$

as appears at once when written out in full. The coefficients $\theta_{i}$ of this relation here appear as the $n$ components of a row vector $\theta$ which is said to annihilate $\Lambda$.

By Smith's Theorem ${ }^{1}$ the matrix $\Lambda$ can be reduced in $\mathcal{F}$ to a diagonal form $D$ such that

$$
H \Lambda K=\operatorname{diag}\left(E_{1}, E_{2}, \ldots, E_{\rho}, 0, \ldots, 0\right)=D
$$

where $H$ and $K$ are nonsingular matrices each of whose elements are homogeneous polynomials in $r, s$, divided possibly by a power of $s$, whereas the determinants $|H|$ and $|K|$ are independent of $r$. The $\rho$ nonzero elements $E$ are the invariant factors. This allows relation (4) to take a simpler form: thus $0=\theta \Lambda=\theta H^{-1} D K^{-1}$. Hence

$$
\phi D=0, \quad \text { where } \phi=\theta H^{-1} .
$$

This $\phi$, so found, is also a row vector: and clearly it can annihilate $D$ if and only if its first $\rho$ components are zero. Thus

$$
\phi=\left[0,0, \ldots, 0, \phi_{\rho+1}, \phi_{\rho+2}, \ldots, \phi_{n}\right]
$$

where the last $\mu(=n-\rho)$ components are arbitrary functions of $r$ and $s$. Let the unit matrix of $n$ rows and columns be written

$$
I=\left\{i_{1}, i_{2}, \ldots, i_{n}\right\}
$$

where $i_{1}=[1,0, \ldots, 0], i_{2}=[0,1, \ldots, 0], \ldots, i_{n}=[0, \ldots, 0,1]$. Then (7) can be written

$$
\phi=\phi_{\rho+1} i_{\rho+1}+\phi_{\rho+2} i_{\rho+2}+\ldots+\phi_{n} i_{n}
$$

1 Turnbull and Aitken : Canonical Matrices (1932), 23. 
which shows that the most general condition (4) is a consequence of $\mu$ linearly independent conditions

$$
i_{\rho+1} D=0, \quad i_{\rho+2} D=0, \ldots, \quad i_{n} D=0 .
$$

Each of these conditions is of the form $i_{h} H \Lambda K=0$, from which the nonsingular $K$ and any scalar common factor of the components can be deleted and the powers of $s$ in the denominators cleared, the result being called $\theta \Lambda=0$. This proves the following theorem.

Theorem I. If the pencil $\Lambda=r A+s B$ possesses row dependence, there are exactly $\mu$ distinct conditions

$$
\theta \Lambda=0
$$

where $\theta$ is a row-vector whose components are homogeneous polynomials in $r$ and $s$ with coefficients in $\mathcal{F},(n-\mu)$ being the rank of $\Lambda$ in $r$ and $s$.

Correlatively: there are exactly $\mu^{\prime}$ distinct relations of column dependence

$$
\Lambda \theta^{\prime}=0
$$

where $\theta^{\prime}$ is a column vector homogeneous in $r$ and $s$.

There is no necessary connection between $\theta$ and $\theta^{\prime}$.

$\S 2$. Let the relations just found be arranged in ascending degree in $r$ and $s$, as

$$
\theta_{1} \Lambda=0, \theta_{2} \Lambda=0, \ldots, \theta_{\mu} \Lambda=0,
$$

where the degree of the veclor $\theta_{i}$ is $m_{i}$, so that

$$
0 \leqq m_{1} \leqq m_{2} \leqq \ldots \leqq m_{\mu}
$$

These are in fact the Kronecker minimal indices of row dependence characterising a singular pencil. A like set $\left[m_{i}^{\prime}\right], \mu^{\prime}$ in number, refers to column dependence. These sets $\left[m_{i}\right]$ and $\left[m_{i}{ }^{\prime}\right]$ are numerical invariants under nonsingular transformation from $\Lambda$ to the type $P \Lambda Q$ above, and also under nonsingular linear transformation from $r, s$ to $r^{\prime}, s^{\prime}$. The proof of these statements is immediate: in either case we have only to suppose the contrary and then obtain an identity of less than minimal order by applying the reciprocal transformation; which involves an absurdity.

These minimal indices together with the set of invariant factors $E_{1}, E_{2}, \ldots, E_{\rho}$ of $\S 1$ (5) completely characterise the pencil $\Lambda$ under such transformations, as Kronecker originally proved. A direct method will now be given for finding these minimal indices. 
§3. Consider the following matrices

$$
\begin{aligned}
& M_{1}=[A, B], M_{2}=\left[\begin{array}{lll}
A & B & . \\
\cdot & A & B
\end{array}\right], M_{3}=\left[\begin{array}{cccc}
A & B & \dot{.} \\
\cdot & A & B & \dot{\cdot} \\
\cdot & A & B
\end{array}\right], \ldots, \\
& N_{1}=\left[\begin{array}{l}
A \\
B
\end{array}\right], N_{2}=\left[\begin{array}{cc}
A & . \\
B & A \\
\cdot & B
\end{array}\right], N_{3}=\left[\begin{array}{ccc}
A & \cdot \\
B & A & \cdot \\
\cdot & B & A \\
. & . & B
\end{array}\right], \ldots,
\end{aligned}
$$

the $M$ consisting of $n, 2 n, 3 n, \ldots$ rows, and the $N$ of $n^{\prime}, 2 n^{\prime}, 3 n^{\prime}, \ldots$ columns respectively. Let $\rho_{i}, \rho_{j}{ }^{\prime}$ denote their respective ranks. Then, if $\mu_{i}=i n-\rho_{i}, \mu_{j}^{\prime}=j n^{\prime}-\rho_{j}^{\prime}$, we have

and

$$
\mu_{1}=n-\rho_{1} \geqq 0, \mu_{2}=2 n-\rho_{2} \geqq 0, \mu_{3}=3 n-\rho_{3} \geqq 0, \ldots,
$$

$$
\mu_{1}^{\prime}=n^{\prime}-\rho_{1}^{\prime} \geqq 0, \mu_{j}^{\prime} \geqq 0 \text {. }
$$

TheOREM 2. If $\mu_{m+1}$ is the first nonzero integer in the sequence $\mu_{1}, \mu_{2}, \ldots$, then $m$ is the value of the smallest minimal index of row dependence, while $\mu_{m+1}$ is the number of such indices which are equal. Column dependence is given similarly by $\mu_{m^{\prime}+1}^{\prime}$.

Proof. By Smith's Theorem, if $\mu_{1}>0$, exactly $\mu_{1}$ distinct relations $\sum_{i} \lambda_{i}$ row $_{i}=0$ exist between the rows of $M_{1}$, where the $\lambda_{i}$ are $2 n^{\prime}$ constants which are not all zero in $\mathcal{F}$. On introducing the row vector

$$
u=\left[\lambda_{1}, \lambda_{2}, \ldots, \lambda_{n}\right] \neq 0
$$

we may put such a relation in the form of a matrix product

$$
u[A, B]=0 \text {, }
$$

that is $u A=0, u B=0$ : so that $u[r A+s B]=0$ for all $r, s$. But $u$ is a nonzero constant vector in $\mathcal{F}$. We have therefore secured a minimal index $m_{1}=0$ : and the number of such is $\mu_{1}(\neq 0)$.

Next if $\mu_{1}=0, \mu_{2}>0$, then a row vector eonsisting of $2 n$ components exists such that

$$
\left[u_{1}, u_{2}\right]\left[\begin{array}{ccc}
A & B & \cdot \\
\cdot & A & B
\end{array}\right]=0, \quad\left[u_{1}, u_{2}\right] \neq 0 .
$$

Here $u_{1}$ is a set of $n$ components, $u_{2}$ is a further set, and in all there are $2 n$ components. Hence

whence

$$
u_{1} A=0, u_{1} B+u_{2} A=0, u_{2} B=0,
$$

$$
\left[u_{1} r+u_{2} s\right][r A+s B]=0 \text {, }
$$


for all values of $r, s$. But this is explicitly a minimal relation $\theta .1=0$, where $\theta=r u_{1}+s u_{2}$ is a vector of index unity. There are $\mu_{2}$ such distinct relations, while there are none of the zero index type, since $u[A, B] \neq 0$ if $\mu_{1}=0$, for all nonzero constant vectors $u$.

Next if $\mu_{1}=0, \mu_{2}=0, \mu_{3}>0$, then (4) and (5) are impossible, but three vectors $u_{1}, u_{2}, u_{3}$ each of $n$ components exist such that

$$
\left[u_{1}, u_{2}, u_{3}\right] M_{3}=0, \quad\left[u_{1}, u_{2}, u_{3}\right] \neq 0 \text {. }
$$

Hence

that is

$$
u_{1} A=0, u_{1} B+u_{2} A=0, u_{2} B+u_{3} A=0, u_{3} B=0 ;
$$

$$
\left[u_{1} r^{2}+u_{2} r s+u_{3} s^{2}\right][r A+s B]=0
$$

for all $r, s$. This gives $\mu_{3}$ distinct relations of index 2. The general case is now evident: it also applies to columns by means of the expressions $\left[\begin{array}{c}A \\ B\end{array}\right]\left\{u_{1}^{\prime}\right\},\left[\begin{array}{cc}A & . \\ B & A \\ \cdot & B\end{array}\right]\left\{u_{1}^{\prime}, u_{2}^{\prime}\right\}$ etc., where $\left\{u_{1}^{\prime}, u_{2}^{\prime}\right\}$ denotes a column of $2 n$ elements. This proves the theorem.

It should be remarked that the matrices $N$ are not the transposed of the $M$ : the elements within $A$ (and $B$ ) maintain their same relative positions. Also, while the method discovers the initial index $m_{1}$ or $m_{1}^{\prime}$ it does not at once discover higher indices, if any.

For example:

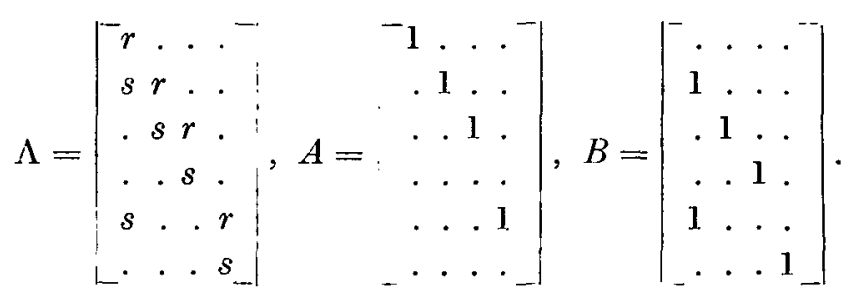

Here the ranks of $M_{1}, M_{2}, M_{3}, M_{4}$ are $6,12,16,20$ respectively so that $\mu_{3}=2$ is the first nonzero $\mu$. This implies two minimal indices each equal to $3-1=2$.

$\S 4$. In the singular case let $m$ denote the smallest minimal index, so that the corresponding minimal relation $\theta \Lambda=0$ can be written more explicitly as

$\left[u_{0} s^{m}-u_{1} s^{m-1} r+u_{2} s^{\prime l-2} r^{2}-\ldots+(-)^{m} u_{m} r^{m}\right](r A+s B)=0$

where each of the $(m+1)$ coefficients $u_{i}$ is a row vector of $n$ constant components. Since this is identically true for all $r, s$ the coefficients 
of powers of $r$ vanish; so that the following minimal chain of vector equations is obtained:

$0=u_{0} B, u_{0} A=u_{1} B, u_{1} A=u_{2} B, \ldots, u_{m-1} A=u_{m} B, u_{m} A=0$.

Theorem 3. If $m$ is the least minimal index of row dependence the vectors $u_{0}, u_{1}, \ldots, u_{m}$ determined by this chain of equations are linearly independent: and so also are the $m$ vectors $u_{1} B, u_{2} B, \ldots, u_{m} B$.

Proof. The theorem is obvious if $m=0$. If $m>0$, the rank $(n-\nu)$ of $B$ must be less than $n$, so that $n$ components for the vector $u_{0}$ can be found (in $v$ distinct ways) satisfying the $n$ scalar equations implied by

$$
u_{0} B=0, \quad u_{0} \neq 0 .
$$

Next the relation $u_{1} A=u_{0} B$ is virtually a set of $n$ nonhomogeneous linear equations to determine the $n$ components of $u_{1}$ in terms of those of $u_{0}$ and the elements of $A$ and $B$. (This step is possible if and only if the rank $n-\nu_{1}$ of $A$ is the same as that of the augmented matrix $\left\{A, u_{0} B\right\}$ which has $(n+1)$ rows. Since the whole chain is already known to exist, at least one of the $v$ values of $u_{0}$ will satisfy this and provide $\nu_{1}$ possible values of $u_{1}$ ).

Let this process be continued for constructing $u_{0}, u_{1}, \ldots, u_{p}$ until $u_{p+1}$ is the first such vector to be linearly related to its predecessors. Then scalar constants $\alpha_{i}$ (zero or otherwise) exist in $\mathcal{F}$ such that

$$
0=u_{p+1}+\alpha_{1} u_{p}+\alpha_{2} u_{p-1}+\ldots+a_{p+1} u_{0} .
$$

Let $(p+1)$ new vectors $v_{i}$ be formed,

$$
\begin{aligned}
& v_{0}=u_{0}, \\
& v_{1}=u_{1}+a_{1} u_{0}, \\
& v_{2}=u_{2}+a_{1} u_{1}+a_{2} u_{0}, \\
& \cdots \ldots \ldots \ldots+\cdots \cdots+a_{p} u_{0}, \\
& v_{p}=u_{p}+a_{1} u_{p-1}+\ldots \ldots+\cdots
\end{aligned}
$$

which are palpably linearly independent, since $u_{0}, \ldots, u_{p}$ are. Then

$$
0=v_{0} B, v_{0} A=v_{1} B, \ldots, v_{p-1} A=v_{p} B, v_{p} A=0
$$

as is at once seen by substituting for each $v$ in terms of the $u_{i}$. For example $v_{0} A-v_{1} B=u_{0} A-\left(u_{1}+a u_{0}\right) B=u_{0} A-u_{1} B=0$. But this is a chain implying a relation $\theta \Lambda=0$ with $u$ replaced by $v(\neq 0)$ and $m$ by $p$. Since $m$ is minimal $m \leqq p$. Hence $u_{0}, u_{1}, \ldots, u_{m}$ are linearly independent since $u_{0}, \ldots, u_{p}$ are. 
For the second part of the theorem let $m>0$. If $u_{q} A$ is the first of the sequence $u_{0} A, u_{1} A, \ldots$ to be linearly dependent upon its predecessors, let

$$
u_{q} A+\beta_{1} u_{q-1} A+\ldots+\beta_{q} u_{0} A=0
$$

or $w_{q} A=0$, where $w_{q}=u_{q}+\beta_{1} u_{q-1}+\ldots+\beta_{q} u_{0}$. By constructing $w_{0}, w_{1}, \ldots, w_{q-1}$ analogously to the $v_{i}$ in (4) it again follows that a chain $0=w_{0} B, \ldots, w_{q} A=0$ exists, which in turn cannot be shorter than the chain (2). Hence $q \geqq m$ : and this proves the theorem.

\section{Reduction to Canonical Form.}

$\S 5$. Consider the following matrix relation

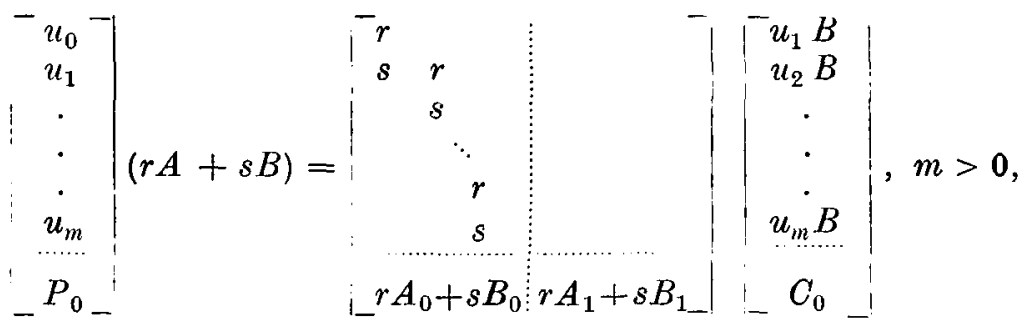

or $P \Lambda=X Q^{-1}$, where $u_{0}, u_{1}, \ldots, u_{m}$ are the first $(m+1)$ rows of $P$. Since by Theorem 3 these are linearly independent, $P$ itself may be made a nonsingular constant matrix by choosing a suitable submatrix $P_{0}$ for its remaining $(n-m-1)$ rows. If $m=0$ then $P \Lambda$ has a zero top row and we pass on to consider lower rows. If $m>0$, then the $m$ rows $u_{i} B$ are also linearly independent, so that a choice of a further submatrix $C_{0}$ is possible, the whole being nonsingular and written $Q^{-1}$. Owing to the chain of relations $u_{i} A=u_{i+1} B$ the first $(m+1)$ rows of the product $P \Lambda$ agree with the corresponding rows of $X Q^{-1}$. For example the $i^{\text {ih }}$ row gives

$$
r u_{i-1} A+s u_{i-1} B=s u_{i-1} B+r u_{i} B .
$$

Hence ( 1$)$ is identically true provided that the remaining $(n-m-1)$ rows of $X$ are identical with those of $P \Lambda Q$. A canonical minimal submatrix $L_{m_{1}}$ of $X$ has now been semi-isolated, such that

$$
X=\left[\begin{array}{c}
L_{m_{1}} \\
r A_{0}+s B_{0}, r \dot{A}_{1}+s B_{1}
\end{array}\right], m=m_{1},
$$

where, for example,

$$
L_{0}=0, L_{1}=\left[\begin{array}{l}
r \\
s
\end{array}\right], L_{2}=\left[\begin{array}{cc}
r & \cdot \\
s & r \\
\cdot & s
\end{array}\right], \ldots,
$$


$m_{1}$ being the lowest index of row dependence. If $X$ contains a second such index $m_{2}$, then $m_{2}$ will in fact be lowest row-index in the submatrix $r A_{1}+s B_{1}$; but it will emerge more directly by selecting a new solution $v_{0}$ of the equation $u B=0$, and forming a new chain (since $\mu>1$ )

$$
v_{0} B=0, v_{1} B=v_{0} A, \ldots, v_{m_{2}} A=0, \quad m_{2} \geqq m_{1} .
$$

THEOREM 4. The $\left(m_{2}+1\right)$ veclors $v$ are linearly independent of themselves and of the $u$ vectors. Also all the vectors $u_{i} B, v_{i} B(i>0)$ are linearly independent.

Proof. Let $v_{p}$ be the first such vector which is linearly dependent upon its predecessors $u$ or $v$. (i) If no vector $u$ with suffix higher than $p$ enters, let the relation be

Construct

$$
0=\sum_{r=0}^{p} \alpha_{r} v_{p-r}+\sum_{r=0}^{p} \beta_{r} u_{p-r}, \quad a_{0}=1 .
$$

$$
\begin{aligned}
& w_{0}=\alpha_{0} v_{0}+\beta_{0} u_{0}, \\
& w_{1}=a_{0} v_{1}+a_{1} v_{0}+\beta_{0} u_{1}+\beta_{1} u_{0}, \text { etc. }
\end{aligned}
$$

exactly as in Theorem 3 . Then the $w$ vectors will form a chain, independent of the $u$ vectors, such that

$$
w_{0} \neq 0, w_{0} B=0, w_{1} B=w_{0} A, \ldots, w_{h} A=0,
$$

where $h=p-1<m_{2}$. This contradicts the assumption. The proof that the $u_{i} B, v_{i} B$ are unrelated is analogous to that in Theorem 3 . (ii) If however terms $u_{q}(q>p)$ enter the relation, write it as

$$
\sum_{r=0}^{p} \alpha_{r} v_{p-r}+\sum_{r=0}^{p} \beta_{r} u_{p-r}=\gamma_{0} u_{q}+\gamma_{1} u_{q+1}+\ldots+\gamma_{m_{1}-\dot{q}} u_{m_{1}}
$$

where $a_{0}=1, \gamma_{0} \neq 0, q>p$. Let $w_{p}$ denote either side of this equality and let $h$ be defined by

$$
p \leqq h=p+m_{1}-q<m_{1} .
$$

From $w_{p}=\gamma_{0} u_{q}+\ldots+\gamma_{m_{1}-1} u_{m_{1}}$ further vectors $w_{p+1}, w_{p+2}, \ldots, w_{h}$ may be derived by successively adding unity to each suffix of $w$ and $u$, and deleting terms of suffix exceeding $m_{1}$. The concluding vector is then $w_{h}=\gamma_{0} u_{m_{1}}$. With those defined by (5) the whole set $w_{0}, w_{1}, \ldots, w_{h}$ is then a chain of index less than $m_{1}$, which again involves a contradiction. The proof that the $u_{i} B, v_{i} B$ are unrelated is. analogous, starting with an identity such as (7) but with $A$ appearing as final factor of each term. Again a chain $w_{0}, \ldots, w_{h}$ would exist, where $w_{0}, \ldots, w_{p}$ are defined by (5), and $w_{p+1}, \ldots, w_{h}$ by the rule just given. This proves the theorem. 
This theorem allows us to take the $v_{i}$ to be the first $\left(m_{2}+1\right)$ rows in $P_{0}$, and the $v_{i+1} B$ the first $m_{2}$ rows in $C_{0}$. The result is

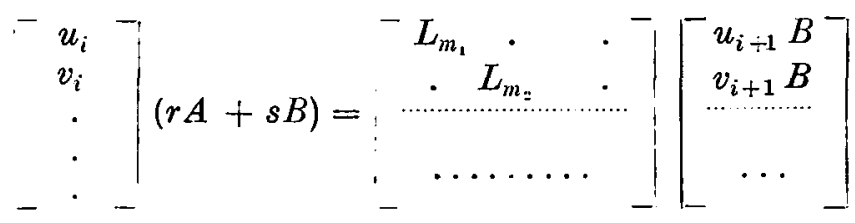

where two canonical minimal submatrices have now been semiisolated. No new feature arises in further steps until all $\mu$ submatrices $L_{m}$ have been semi-isolated. Among themselves they are completely isolated in the form

$$
L=\operatorname{diag}\left(L_{m_{1}}, L_{m_{2}}, \ldots, L_{m_{\mu}}\right) .
$$

This exhausts all possible row dependence. Column dependence is then sought in $\mu^{\prime}$ possible ways, but owing to the isolation of each $L_{m_{i}}$ in its own row, such column dependence is independent of columns occupied by $L$. The result is a submatrix

$$
L^{\prime}=\operatorname{diag}\left(L_{m_{1}^{\prime}}^{\prime}, \ldots, L_{m_{\mu^{\prime}}^{\prime}}^{\prime}\right)
$$

and any further submatrix $X_{0}$ not lying in the rows or columns of $L$ and $L^{\prime}$ must be nonsingular. This can be reduced to rational or classical canonical form $S$ say, and finally all remaining nonzero elements other than those of $L, L^{\prime}$ and $S$ can be removed by the methods earlier explained. ${ }^{1}$

§6. Also, for directly obtaining the rational form of the nonsingular portions of the pencil, vector chains of the same general type $u_{i+1} A=u_{i} B$ may be formed but for which $u_{0} \neq 0, u_{0} A \neq 0,|A| \neq 0$. They must then be examined in descending order of their length, as in the rational case ${ }^{2}$ for the collineatory group. The method is sufficiently illustrated by the following example:

$$
\left[\begin{array}{l}
u_{0} \\
u_{1} \\
u_{2}
\end{array}\right](r A+s B)=\left[\begin{array}{cll}
r, & s, & \\
& r, & s \\
a_{0} s, & a_{1} s, & r+a_{2} s
\end{array}\right]\left[\begin{array}{l}
u_{0} A \\
u_{1} A \\
u_{2} A
\end{array}\right] .
$$

In this example $u_{3}=a_{0} u_{0}+a_{1} u_{1}+a_{2} u_{2}$ is the first of such a chain to be related to its predecessors.

1 Canonical Matrices (1932), 127-8.

2 Canonical Matrices, 49. 
It is to be noted that in the example of $\S 3(9)$ the chain appearing in the first four rows of $\Lambda$ is not a true minimal. The failure is due to the presence of the lower element $s$ in the first column. Every vector satisfying $u_{0} B=0$ must be of the form

$$
[a, \beta, 0,0,-\beta, 0] \text {, }
$$

where $\alpha, \beta$ are arbitrary constants. Taking $a=1, \beta=0$, the shortest chain is obtained as

$$
u_{0}=[1,0,0,0,0,0], u_{1}=[0,0,0,0,1,0], u_{2}=[0,0,0,0,0,1]
$$

where $u_{0} B=u_{0} A-u_{1} B=u_{1} A-u_{2} B=u_{2} A=0$.

It may also be noted that the same method will furnish every submatrix of type

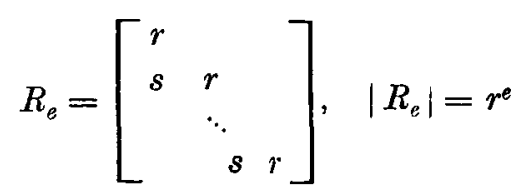

due to a zero latent root, and belonging to the nonsingular core. All such are found according to ascending value of $e$ by use of every vector $u_{0}$ for which $u_{0} B=0$ but which does not lead to a minimal chain. A modified chain now appears, following the same law except that it terminates abruptly with $u_{e-1}$ at a point where it is impossible to satisfy the equation $u_{e} B=u_{e-1} A$ by any vector $u_{e}$. 